\title{
Does the mass distribution in discs influence encounter-induced losses in young star clusters?
}

\author{
Manuel Steinhausen and Susanne Pfalzner
}

\author{
Max-Planck-Institut für Radioastronomie, Auf dem Hügel 69, 53121 Bonn, Germany \\ e-mail: mstein@mpifr.de
}

Received 25 November 2013 / Accepted 5 March 2014

\begin{abstract}
Context. One mechanism for the external destruction of protoplanetary discs in young dense clusters is tidal disruption during the flyby of another cluster member. The degree of mass loss in such an encounter depends, among other parameters, on the distribution of the material within the disc. Previous work showed that this is especially so in encounters that truncate large parts of the outer disc. The expectation is that the number of completely destroyed discs in a cluster also depends on the mass distribution within the discs. Aims. Here we test this hypothesis by determining the influence of encounters on the disc fraction and average disc mass in clusters of various stellar densities for different mass distributions in the discs.

Methods. This is done by performing NBODY6 simulations of a variety of cluster environments, where we track the encounter dynamics and determine the mass loss due to these encounters for different disc-mass distributions.

Results. We find that although the disc-mass distribution has a significant impact on the disc losses for specific star-disc encounters, the overall disc frequency generally remains unaffected. The reason is that in single encounters the dependence on the mass distribution is strongest if both stars have very different masses. Such encounters are rather infrequent in sparse clusters. In dense clusters these encounters are more common; however, here the disc frequency is largely determined by encounters between low-mass stars such that the overall disc frequency does not change significantly.

Conclusions. For tidal disruption the disc destruction in clusters is fairly independent of the actual distribution of the material in the disc. The all determining factor remains the cluster density.
\end{abstract}

Key words. protoplanetary disks - stars: protostars - circumstellar matter - open clusters and associations: general

\section{Introduction}

Young stars are initially surrounded by a circumstellar disc. Observations show that with time the circumstellar discs in young clusters become depleted of gas and dust and eventually disappear. It is currently unclear which physical mechanism dominates the evolutionary disc destruction processes. Among the great variety of effects are internal processes such as viscous torques (e.g. Shu et al. 1987), turbulent effects (Klahr \& Bodenheimer 2003), and magnetic fields (Balbus \& Hawley 2002), but also external disc destruction processes like photoevaporation (Scally \& Clarke 2001; Clarke et al. 2001; Matsuyama et al. 2003; Johnstone et al. 2004; Alexander et al. 2005, 2006; Ercolano et al. 2008; Drake et al. 2009; Gorti \& Hollenbach 2009) and tidal interactions (Heller 1993; Hall et al. 1996; Clarke \& Pringle 1993; Ostriker 1994; Heller 1995; Hall 1997; Larwood 1997; Boffin et al. 1998; Pfalzner 2004; Pfalzner et al. 2005a; Moeckel \& Bally 2006; Kley et al. 2008).

In this study we investigate the early embedded phase of stellar cluster development with the scope to understand the general influence of gravitational interactions on the disc frequencies. In contrast to previous investigations (Clarke \& Pringle 1993; Hall et al. 1996; Boffin et al. 1998; Pfalzner 2004; Pfalzner et al. 2005a; Olczak et al. 2006; Moeckel \& Bally 2006; Kley et al. 2008) we concentrate on the influence of the mass distributions in the disc on the resulting disc frequency.

Because of the temperature and dust grain size distribution in discs, infra-red observations can only resolve the inner areas $(<10 \mathrm{AU})$. Alternatively, observations in the submillimetre range are limited to the outerskirts (50 AU). So no continuous observational spatial coverage of entire discs with high resolution exist to date. Nevertheless, by fitting resolved millimetre continuum or line emission data with parametric disc structure models (e.g. Mundy et al. 1996; Lay et al. 1997) a wide variety of different surface densities profiles have been derived. Another method is the combination with broadband spectral energy distributions (SEDs; Wilner et al. 2000; Testi et al. 2001; Akeson et al. 2002; Kitamura et al. 2002; Andrews \& Williams 2007). These studies have profoundly shaped our knowledge of disc structures; however, all have fundamentally been limited by the low angular resolution of available data. Thus, to date there is no conclusive knowledge about the typical surface density of protoplanetary discs and how it develops with time.

Most previous numerical studies of star-disc encounters used a theoretically motivated $r^{-1}$-dependent disc-mass distribution (Hall et al. 1996; Hall 1997; Pfalzner 2004; Olczak et al. 2006; Moeckel \& Bally 2006; Pfalzner \& Olczak 2007). Recently, Steinhausen et al. (2012) investigated for a wide parameter space the relative disc-mass loss in encounters with special focus on the dependence on the mass distribution in the disc and found differences of up to $40 \%$ between the different initial density distributions for the same type of encounter. The aim of this study is to investigate whether this means a comparable difference in the encounter-induced disc frequency in clusters.

The likelihood of encounters is a function of the stellar density which varies between different clusters but also within the considered clusters. To first order the encounter frequency, $\eta$, 
depends on the stellar number density, $n_{\text {star }}$, as $\eta \propto n_{\text {star }}$ (Binney $\&$ Tremaine 2008). This approximation is valid for systems with equal-mass stars undergoing two-body encounters. For systems with unequal stellar masses effects like gravitational focusing become important (Binney \& Tremaine 2008) and for very high stellar densities the two-body approach breaks down (Pfalzner \& Kaczmarek 2013). The cluster-average densities span a wide range from sparse clusters like Taurus with densities of 1-10 stars per $\mathrm{pc}^{3}$ (Luhman et al. 2009) to very massive and compact clusters like the Arches cluster with a core density of several $10^{5}$ stars per $\mathrm{pc}^{3}$ (Figer et al. 1999). Such dense stellar environments lead to strong gravitational interactions between the cluster members and affect also the protoplanetary discs.

The mass (and angular momentum) losses due to gravitational interactions in clusters as a function of stellar densities has been investigated in several studies (Pfalzner 2004; Olczak et al. 2006; Adams et al. 2006; Pfalzner \& Olczak 2007). In particular, Olczak et al. (2006) found that star-disc interactions influencing the circumstellar discs are more frequent than previously assumed (Scally \& Clarke 2001). Not only does the encounter frequency depend on the stellar density, but also on the prevalent type of encounter. In sparse clusters distant parabolic two-body encounters dominate, whereas in dense clusters close encounters often involving several stars at once become increasingly important (Olczak et al. 2010).

Observations confirm that the cluster disc fraction (CDF) depends strongly on the stellar cluster density. For example, Luhman et al. (2008) found a significantly higher number of dissolved discs in the dense IC 348 cluster compared to the sparse Chamaeleon I cluster of the same age. Similarly, Stolte et al. (2010) detected a strong decrease in the disc fraction close to the dense centre of the Arches cluster, one of the densest stellar populations in the Milky Way $\left(\rho_{\text {core }}>10^{5} M_{\odot} \mathrm{pc}^{-3}\right.$. Similar results have been obtained for the starburst cluster NGC 3603 (Stolte et al. 2004) and the Orion nebula cluster (ONC, Hillenbrand et al. 1998). In addition, observed disc frequencies in sparse stellar associations show a slower decrease than in denser clusters (Fang et al. 2013). However, these observations have not been able to distinguish whether encounters or photo-evaporation is the dominant environmental process of disc destruction.

In the following we will address how the mass distribution in protoplanetary discs influences encounter-induced losses in young stellar clusters. First, the method and the setup parameters are detailed in Sect. 2. In Sect. 3 the influence of a varying initial disc-mass distribution on the encounter-induced losses in a stellar cluster is presented. Finally, a discussion will be given in Sect. 4 and the results will be summarised in Sect. 5 .

\section{Method}

First, we simulated the dynamical interactions between all stars in typical stellar clusters for several Myr of cluster evolution. We use the code NBODY6 (Aarseth 1963, 1974, 2003), which has been modified by adding an encounter tracking routine (see Olczak et al. 2012, for more details). For each encounter event the encounter mass ratios and periastron distances are tracked and used to determine the mass loss in the discs. We used the recent results of Steinhausen et al. (2012) to determine this mass loss for various mass distributions within the discs.

\subsection{Cluster models}

In the $N$-body simulations we chose the masses of the stars in the cluster according to the initial mass function (IMF) given by
Kroupa (2001). Stellar masses below the hydrogen burning limit $\left(m_{\text {star }}<0.08 M_{\odot}\right)$ are neglected since they contribute very little to the stellar dynamics (Allen et al. 2005).

In our simulations all stars are assumed to be initially single. Primordial binaries have been neglected for two reasons: first, using only single stars reduces the computational cost in the cluster simulations and, second, treating the mass loss in the disc involving three stars would add another set of variables to the already extensive parameter study of encounters. However, during the cluster evolution a small fraction of binaries form via capture processes. Further encounters of these systems are excluded from the disc-mass loss (Pfalzner \& Olczak 2008). The effect of primordial binaries on the results for the initial discmass distributions will be discussed in Sect. 4.

The frequncy of star-disc encounters in a young cluster is largely determined by its stellar number density distribution. The ONC is regarded as the prototype of an embedded dense cluster. Observations of today's ONC density distribution show that it can be approximated by an isothermal profile $\left(\propto r^{-2}\right)$ in the outer parts (McCaughrean \& Stauffer 1994; Hillenbrand \& Hartmann 1998) and a flat stellar density profile of the form $\rho_{\text {core }} \propto r^{-0.5}$ in the cluster core (Scally et al. 2005). Here, an isothermal density model with an initially slightly increased density in the cluster core region is used, given by

$\rho_{\text {initial }}(r)=\left\{\begin{array}{cl}\rho_{0} \cdot r^{-2.3} & r \leq R_{\text {core }} \\ \rho_{0} \cdot r^{-2.0} & R_{\text {core }}<r \leq R_{\text {cluster }} \\ 0 & R_{\text {cluster }}<r\end{array}\right.$

where $\rho_{0}=3.1 \times 10^{2} \mathrm{pc}^{-3}, R_{\text {core }}=0.2 \mathrm{pc}$, and $R_{\text {cluster }}=2.5 \mathrm{pc}$. This distribution develops within $1 \mathrm{Myr}$, the estimated age of the ONC, to the stellar distribution observed for the ONC today (Olczak et al. 2006). Following the approach by Bonnell \& Davies (1998), initially the four most massive stars have been placed in the inner cluster region with $r_{\text {seg }}=0.6 \cdot r_{\mathrm{hm}}$, where $r_{\mathrm{hm}}$ is the half-mass radius of the cluster.

We model clusters of different density by keeping the size of the cluster constant ( $\left.r_{\text {cluster }}=2.5 \mathrm{pc}\right)$ and varying the initial number of stars $N_{\text {stars }}$. The covered density range here is representative of massive clusters in the solar neighbourhood in the late embedded stage. Using a slightly steeper profile of $\rho_{\text {core }}(r) \propto r^{-2.3}$ in the cluster core has only a minor effect.

For our simulations of embedded clusters we use the standard method of no gas being included. Since we are in particularly interested in the effect of stellar encounters rather than the cluster evolution, the focus here will be on initially virialised systems of $Q=0.5$. The stellar velocities are chosen accordingly. To model the stellar velocities, a Maxwellian velocity distribution with radius-independent velocity dispersion $\sigma$ has been used. Despite being in virial equilibrium the cluster still expands to some degree, so that the stellar density is highest in the earliest stages of the development.

It is indispensable to perform multiple simulations $\left(N_{\text {sim }}\right)$ for each individual cluster model to obtain statistically robust results. Therefore, a set of random initial stellar positions, velocities, and masses has been achieved for each run, which are analysed and averaged in a subsequent step. It turned out that tracking at least 500000 stellar trajectories (Pfalzner \& Kaczmarek 2013) leads to an error of $<3 \%$ for each of the presented results. For an initial number of stars $N_{\text {star }}=1000$, around 500 simulations were performed while 16 simulations were performed when $N_{\text {star }}=32000$.

An overview of the parameters of the simulated models, like initial number of stars $N_{\text {stars }}$ and corresponding number of 
Table 1. Number of initial stars $N_{\text {stars }}$, number of performed simulations $N_{\text {sim }}$, number density of the cluster centre region $\rho_{\text {centre }}$, and total cluster density $\rho_{\text {cluster }}$ for the six investigated models.

\begin{tabular}{lcccc}
\hline \hline Model & $N_{\text {stars }}$ & $N_{\text {sim }}$ & $\rho_{\text {centre }}\left[10^{3} \mathrm{pc}^{-3}\right]$ & $\rho_{\text {cluster }}\left[\mathrm{pc}^{-3}\right]$ \\
\hline A & 1000 & 500 & 1.3 & 15.3 \\
B & 2000 & 250 & 2.7 & 30.6 \\
C & 4000 & 125 & 5.3 & 61.1 \\
D & 8000 & 70 & 10.5 & 122.2 \\
E & 16000 & 32 & 21.1 & 244.5 \\
F & 32000 & 16 & 42.0 & 489.2 \\
\hline
\end{tabular}

Notes. The radius of the core region is $r_{\text {centre }}=0.3 \mathrm{pc}$, while the cluster size is $r_{\text {cluster }}=2.5 \mathrm{pc}$. The core density is given by $\rho_{\text {centre }}=$ $3 N_{\text {centre }} / 4 \pi r_{\text {centre }}^{3}$ and the cluster density by $\rho_{\text {cluster }}=3 N_{\text {stars }} / 4 \pi r_{\text {cluster }}^{3}$.

simulations $N_{\text {sim }}$, is shown in Table 1 , as well as the resulting densities in the cluster centre region $\rho_{\text {centre }}$ (with $r_{\text {centre }}=0.3 \mathrm{pc}$, e.g. the radius of the Trapezium cluster) and the mean cluster density $\rho_{\text {cluster }}$ within the cluster radius $r_{\text {cluster }}=2.5 \mathrm{pc}$. For details about the dynamical evolution of the simulated clusters see Olczak et al. (2010, Figs. 2 and 3 for the temporal development of the surface density profile and the cluster disc fraction, respectively).

\subsection{Disc-mass loss}

During the cluster simulations for each encounter, the mass ratio and the periastron are tracked. Only afterwards, in the diagnostics part, the respective mass losses for all encounter events are determined. These losses depend on the mass distribution in the disc before the encounter. Here the tabulated data detailed in (Steinhausen et al. 2012) are used, where first-order interpolations were applied for the values between the tabulated data. These data (see Steinhausen et al. 2012, for more details) are for coplanar, parabolic encounters. As such they represent upper limits for the disc-mass losses.

Initially all stars are surrounded by a disc of size $r_{\text {disc }}=$ $150 \mathrm{AU}$. This value represents an average value of observed disc sizes. Here we investigate different power-law mass distributions of the disc material of the form

$\Sigma(r) \propto r^{-p}$.

This corresponds to the standard form used for fitting in observations. Observationally determined indices $p$ range roughly from $p=0$ to $p=2$ (see Steinhausen et al. 2012, for more details). Here we treat specifically the cases $p=0, p=1$, and $p=7 / 4$.

\section{Results}

In the following the focus will be on the central cluster regions $\left(r_{\text {centre }}=0.3 \mathrm{pc}\right)$, representing the densest part of the stellar population. Here a large fraction of stars is involved in encounter events, which makes it easier to spot the influence of the different mass distributions. The simulations cover only the first $2 \mathrm{Myr}$ of cluster development because the embedded phase of clusters lasts typically 1-3 Myr.

Several studies demonstrated that the typical kind of encounter depends strongly on the cluster density (Olzcak et al. 2010, 2012; Dukes \& Krumholz 2012). In low-density clusters gravitational focussing by the massive stars dominates the disc destruction process, whereas in very dense clusters the interactions between low-mass stars become important.

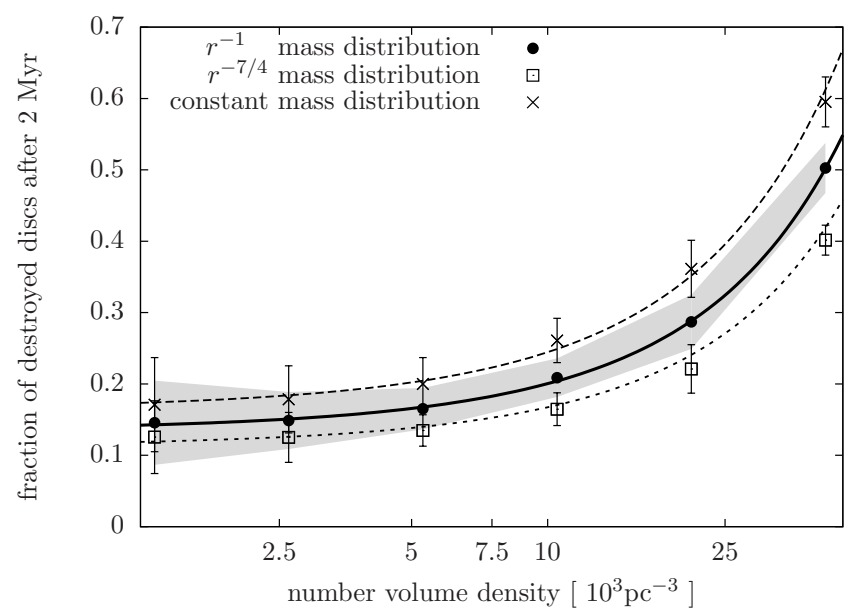

Fig. 1. Fraction of destroyed discs after $2 \mathrm{Myr}$ of evolution as a function of the stellar number volume density in the central cluster region $\left(r_{\text {centre }}=0.3 \mathrm{pc}\right)$. Circles represent the results of an initial $r^{-1}$ discmass distribution, while crosses show the constant and open squares the $r^{-7 / 4}$ distributions. The lines represent a smooth Bezier fit to the data points. The filled curve indicates the standard deviation for $p=1$, which is of the same order for the other distributions as indicated by the error bars.

\subsection{Cluster disc fraction}

Figure 1 shows the fraction of destroyed discs, $F_{\text {destroyed, }}$ after $2 \mathrm{Myr}$ of cluster evolution as a function of the central cluster densities in the range $1 \times 10^{3}$ to $4 \times 10^{4} \mathrm{pc}^{-3}$ (represented by Model A-F). For initial disc-mass distributions of $r^{-1}$ (filled circles) central cluster densities of $10^{3} \mathrm{pc}^{-3}$ lead to around $15 \%$ of all circumstellar discs being destroyed, while for volume densities above $10^{4} \mathrm{pc}^{-3}$ the fraction of destroyed discs increases considerably to up to $50 \%{ }^{1}$. It can be seen that the general trend is the same for different initial mass distributions in the disc.

Using an initial disc-mass distribution that differs from the $r^{-1}$ case, the maximum relative difference in the fraction of destroyed discs to the standard case of a $r^{-1}$ distribution is about $25 \%$. Since in general more discs are tidally destroyed in dense clusters, one obtains that the higher the cluster density, the larger the absolute differences in the fraction of destroyed discs for the different mass distributions. Here, an initially constant disc-mass distribution provides a maximum for the encounterinduced disc-mass losses, because material from the outer disc parts is removed.

In other words, for low and intermediate densities, $\rho_{\text {centre }} \leq$ $10^{4} \mathrm{pc}^{-3}$, the cluster disc fractions for the different initial distributions are found to differ only slightly from the results found for an $r^{-1}$ distribution and are most likely not observationally detectable with current number statistics. However, in the case of $\rho_{\text {centre }}=4 \times 10^{4} \mathrm{pc}^{-3}$ (Model $F$ ) for an initially constant discmass distribution, most circumstellar discs $(60 \%)$ are destroyed by encounters, whereas for the $r^{-7 / 4}$ distribution the fraction is only about $40 \%$. This means that in very dense clusters, where the total fraction of destroyed discs increases significantly, the initial disc-mass distribution might be important.

How do the differences between the cluster disc fractions depend on the stellar masses involved? Fig. 2 shows the fraction of

1 The fraction of destroyed discs is slightly lower than in Olczak et al. (2010) because they used a fit formula for the disc-mass losses that slightly overestimates the losses in the case of mass ratios $M_{2} / M_{1}>20$. Here, an interpolation algorithm based on an extended parameter study is used. 



Fig. 2. Fraction of destroyed discs after $2 \mathrm{Myr}$ as a function of the stellar mass for a) Model A and b) Model F. The results for an initially constant disc-mass distribution are shown as grey bars and the $r^{-7 / 4}$ case is indicated by black bars. The average deviation per mass bin is $<5 \%$. Stellar masses $>50 M_{\odot}$ have been excluded because of a low number of stars in these mass regimes and larger errors of up to $10 \%$.

destroyed discs as a function of the stellar mass for Model A (Fig. 2a) and Model F (Fig. 2b). It can be seen that again for sparser clusters (Fig. 2a) differences between an initially constant and an $r^{-7 / 4}$ disc-mass distribution on the stellar masses are of minor importance as they are usually well below 5\%. Less than $20 \%$ of low-mass stars lose their discs, while for high-mass stars the discs are generally completely destroyed by multiple interactions with the surrounding stars.

For dense clusters (Fig. 2b), by contrast, the relevance of the initial disc-mass distribution depends strongly on the mass of the disc-surrounded star. For stellar masses $M_{1}>2 M_{\odot}$ the differences in the fraction of disc-less stars usually remain below $\ll 10 \%$, even though up to $80 \%$ of circumstellar discs are destroyed. The reason lies in the encounter mass ratios, $M_{2} / M_{1}$. Independent of the cluster density, for large stellar masses the median encounter mass ratio is $M_{2} / M_{1} \leq 1$. For such low mass ratios the differences between the disc losses for the various discmass distributions is small (see Steinhausen et al. 2012).

By contrast, high encounter mass ratios are obtained for lowmass stars $M_{1}<2 M_{\odot}$, where the median encounter mass ratio is typically $M_{2} / M_{1}>10$ in dense clusters. In the case of $M_{1}<2 M_{\odot}$ the differences between the losses for the different initial disc-mass distributions increase to about $23 \%$. Since most of the cluster members are low-mass stars, the differences



Fig. 3. Fraction of stars with perturbed discs after $2 \mathrm{Myr}$ of evolution as a function of the stellar number volume density in the central cluster region $\left(r_{\text {centre }}=0.3 \mathrm{pc}\right)$. Circles represent the results of an initial $r^{-1}$ discmass distribution, while crosses show the constant and open squares the $r^{-7 / 4}$ distributions. The line represents a smooth Bezier fit to the data points for the $r^{-1}$ distribution. The error bars indicate the standard deviation for $p=1$, which is of the same order for the other distributions.

between the total cluster disc fractions is dominated by the fraction of discs around these low-mass stars. As in very dense clusters, low-mass stars contribute significantly to the disc destruction process and the overall disc-mass losses increase, while at the same time the differences between the investigated disc-mass distributions become more pronounced.

\subsection{Disc properties}

In Sect. 3.1 the focus was on strong perturbations that lead to complete disc destruction. These destructive encounters are relatively rare events. Much more common are weaker stellar encounters; these do not lead to disc destruction, but can have a significant influence on the disc properties, such as total disc mass, disc size, and the mass distribution within the disc. This process is often overlooked, but it might be of major importance for the properties of the potentially forming planetary system.

Next we will include these weaker interactions in our investigation to determine their general influence on the disc properties. We take into account any encounter that leads to a $\sim 5 \%$ change in the disc's angular momentum. This particular value was chosen because in this case the complete disc mass generally remains bound to the disc-surrounded star even though a significant perturbation of the disc outskirts happens (Pfalzner 2003; Pfalzner \& Olczak 2007). In the following these discs are referred to as perturbed discs.

Figure 3 shows the fraction of stars with perturbed discs after $2 \mathrm{Myr}$ as a function of the central cluster density. We find that the fraction of stars with perturbed discs increases constantly from $40 \%$ for clusters with $\rho_{\text {centre }}=1 \times 10^{3}$ to $95 \%$ for central densities of $4 \times 10^{4} \mathrm{pc}^{-3}$. In dense clusters almost every star experiences an encounter event within the first $2 \mathrm{Myr}$ in the dense inner cluster regions ${ }^{2}$.

2 The standard deviation for sparse clusters is found to be up to $7 \%$, while the deviations for dense clusters are only $<1 \%$. In sparse clusters disc losses are specified by the few high-mass stars and depend strongly on their position within the cluster. In dense clusters an increasing number of low-mass stars is involved in encounter events reducing the prominent effect of the high-mass stars. 




Fig. 4. Average number of encounters per disc-less star after 2 Myr of evolution is shown as a function of the mass of the central star for Model A (dashed grey) and Model F (solid black). A constant initial disc-mass distribution was used since the number of encounters deviates only slightly for the different investigated initial disc-mass distributions. The average deviation per mass bin is $<3 \%$.

As weak, distant encounters are much more common than strong ones, one would expect a difference between the different disc-mass distributions for the number of stars that experience a perturbing encounter. Figure 3 also shows the results for a constant initial disc-mass distribution (crosses) and a $r^{-7 / 4}$ distribution (open squares). Surprisingly, the fraction of stars with perturbed discs is basically the same for all initial disc-mass distributions. In sparse clusters the encounter statistics are dominated by interactions with the massive stars in the cluster centre; these stars act as gravitational focii for the low-mass stars and usually strongly perturb their stellar discs. By contrast, in dense clusters multiple interactions between the low-mass stars result in nearly all discs being strongly perturbed. In total, the differences in the fraction of perturbed discs between the investigated initial disc-mass distributions are minor.

As mentioned before, despite the lower encounter mass ratios in the high-mass star regime the fraction of destroyed discs remains high because of multiple encounter events. Here again the answer lies in the number of perturbing encounters. Figure 4 shows the average number of encounters per disc-less star as a function of the mass of the central star after $2 \mathrm{Myr}$ for the inner cluster region. Whereas in sparser clusters (Fig. 4, light grey) low-mass stars usually experienced less than ten encounters per star, this number increases significantly for high-mass stars. On average the most massive stars $\left(M_{1}>50 M_{\odot}\right)$ undergo more than 500 encounters.

For dense clusters, like Model F (Fig. 4, black), the situation is different: the number of encounters for low- and intermediate-mass stars increases significantly. Gravitational focusing becomes less important and the number of encounters for high-mass stars decreases. The result is a nearly equal number of encounters for low- to intermediate mass stars. Each star experiences $\sim 30$ encounter events. The exceptions are a few of the most massive stars that experience fewer encounters than in lowdensity clusters, but still about ten times more than the rest of the stars with destroyed discs.

\section{Discussion}

The results presented here can be regarded as upper limits for the influence of encounters on protoplanetary discs in the different stellar environments. One reason is that in above calculations each star was assumed to be initially surrounded by a disc and at the same time only encounters with a disc-less perturbing star were investigated. However, Pfalzner et al. (2005a) showed that star-disc encounter results can be generalised to disc-disc encounters as long as there is no significant mass exchange between the discs. In the case of close encounters the discs might be replenished to some extent, which would lead to an overestimate of the losses in strong perturbing encounters. Most affected by this simplification would be discs with shallow mass distributions, which would be replenished to a greater degree. However, the consequence would be an even smaller difference between the fraction of destroyed discs for the various mass distributions. So our general result, that the disc-mass distribution has little effect on the disc fraction, would still hold.

Another simplification is the focus on prograde, coplanar encounters. If the cluster is not fast rotating, an alignment of the disc and the encounter plane seems rather unlikely. However, as long as the inclination is not larger than 45 degrees, the disc losses due to inclined encounters are only slightly reduced in comparison to a coplanar encounter (Pfalzner et al. 2005b). Hence, if the orientations were completely randomly distributed the losses would be overestimated in $75 \%$ of the encounters. This would be the same for any of the considered mass distributions.

Similarly, it has been assumed that the relative losses remain unchanged in a consecutive encounter. Pfalzner (2004) showed that for equal-mass perturbers a second encounter results not in the same absolute, but in the same relative losses as in the first encounter (see also Tackenberg 2009). However, since the perturbations also lead to a steeper surface density profile of the remaining discs, our treatment of repeated encounters might lead again to an overestimate of the losses.

Additionally, encounters have been generally treated as parabolic $(\epsilon=1)$ throughout this work. For hyperbolic encounters, $\epsilon>1$ ), because of the shorter interaction times, the total disc losses would drop considerably. In sparse clusters these hyperbolic encounters can be generally neglected. In contrast, the average eccentricity of the stellar orbits is higher in the case of dense stellar environments, where the massive stars lose their dominating role as encounter partners (Olczak et al. 2010). All mass distributions should be affected to the same degree by this simplification.

Another significant factor in disc losses is the initial disc size. Small disc sizes lead to higher relative periastron distances $r_{\text {peri }}=r / r_{\text {disc }}$ and therefore lower levels of disc loss in our calculations. In this context, observations give no clear picture, providing a multitude of observed disc-mass distributions and sizes. Thus, here the discs are assumed to have a radius of $r_{\text {disc }}=150 \mathrm{AU}$, which is a typical observed value. However, a scaling of the disc size with the mass of the disc-surrounded star by $r_{\text {disc }}=150 \mathrm{AU} \cdot \sqrt{M_{1}\left[M_{\odot}\right] / M_{\odot}}$, as is obtained if a fixed force of the stars at the disc's outer radius is assumed, would be equally likely. This would result in an increased disc diameter for massive stars $\left(m_{\text {star }}>1 M_{\odot}\right)$ while the disc sizes of low-mass stars $\left(m_{\text {star }}<1 M_{\odot}\right)$ are significantly reduced. In consequence this would lead to a lower fraction of destroyed discs, since the most of the stars in the cluster are located in the lowmass regime.

All these simplifications might lead to overestimating the levels of disc loss. However, some of the applied assumptions 
potentially lead to an underestimation. First of all, substellar objects $\left(M_{\text {star }}<0.08 M_{\odot}\right)$ have been excluded in the present study. In general, the mass ratios in encounters with such low-mass objects are well below 0.1 , which implies that the disc losses would be sufficiently small $(<10 \%)$. Hence, the effect should be minor for massive stars. If the encounter is non-penetrating the effect can be neglected even for low-mass stellar objects.

Furthermore, all encounter processes have been treated as two-body encounters. Umbreit (2001) showed that multiplebody encounters result in higher levels of disc loss. However, the effect strongly depends on the mass and periastron distance of the involved stars. For the present calculations the most destructive encounter has been used to obtain the disc losses, while the other encounter partners are most likely either distant or less massive, so that their influence on the losses is less significant.

Primordial binaries have not been included, which might significantly underestimate the destruction rates of stellar discs especially for tight binaries. While it is suggested that up to $100 \%$ of all stars (e.g. Kroupa 1995, and references therein) might be initially part of a binary system, it remains unclear how their initial periods are distributed. Assuming the upper limit case of an initially log-uniform period distribution (e.g. Reipurth et al. 2007) a fraction of $50 \%$ of all stars would have had a companion with a semi-major axis $\leq 100 \mathrm{AU}$. Apart from an increased destruction rate of the stellar discs in tight binaries, the cluster dynamics are usually influenced by strong few-body interactions (Hills 1975; Heggie 1975), which potentially leads to underestimating the number of ejections from the cluster. Hence, primordial binaries might have a non-negligible effect on the disc fractions and stellar dynamics and further investigations are needed to give an estimate of the effect.

Finally, a large fraction of disc-less stars is ejected after an encounter event within the first few $10^{5} \mathrm{yr}$ with velocities larger than $20 \mathrm{pc} / \mathrm{Myr}$, populating regions of $>20 \mathrm{pc}$ distance from the cluster centre. In the context of planet formation such stellar high-velocity escapers from embedded clusters are expected to show no excess infrared emission and to be less frequently surrounded by planets. Similar results have been obtained from observational (Hillenbrand 1997; Lada et al. 2004) and numerical studies that assumed primordial mass segregated populations (Olczak et al. 2008). However, such high-velocity escapers are less frequent for primordial non-mass segregated clusters. Here, a first approach showed that in contrast to evenly distributed clusters, in mass-segregated clusters a lower fraction of disc-less stars remains in the core region as more stars are ejected due to gravitational focusing of the high-mass stars. The consequence is an increased fraction of disc-less stars in the core region of primordial non-mass segregated clusters of up to $10 \%$ in the tested extreme cases.

\section{Summary}

The focus in this work was on the question of how far the mass distribution within a protoplanetary disc influences the rate of tidally destructed discs in typical cluster environments. Stellar clusters spanning a large range of densities have been modelled and the influence of these different environments on the disc fraction has been investigated. The main results are as follows.

1. Surprisingly, even though the initial disc-mass distribution significantly influences individual disc losses induced by stellar interactions, the fraction of discs that are completely destroyed by encounters remains fairly unaffected, as long as the cluster density does not exceed $\rho_{\text {core }}<10^{4} \mathrm{pc}^{-3}$. These are still quite dense clusters, for example the ONC would belong to this group. The reason is that in these clusters the complete destruction of discs happens by interactions with high-mass stars. This type of encounter is rather insensitive to the initial disc-mass distribution.

2. By contrast, for very dense clusters, an example would be NGC 3603, the fraction of destroyed discs depends to some degree on the initial disc-mass distribution. More specifically, $60 \%$ of discs are destroyed assuming initially constant disc-mass distributions while for an initially steep disc-mass distribution $\left(\Sigma \propto r^{-7 / 4}\right)$ only $40 \%$ are affected. In such dense clusters, interactions between low-mass stars are not only more frequent in absolute terms but also in relative terms. Such encounters between low-mass stars show the strongest dependence on the mass distribution in the disc. However, in general even in this case the fraction of destroyed discs deviates no more than $\sim 20 \%$ from an initially $r^{-1}$ disc-mass distribution.

3. The initial disc-mass distribution has little influence on the total number of stars that have a disc that is changed in its structure by a fly-by. However, independently of the initial distribution of the disc material, almost all circumstellar discs $(95 \%)$ in the core region $\left(r_{\text {core }}\right)$ of dense clusters are significantly effected by fly-bys.

This means the most results usually obtained assuming a $r^{-1}$ disc-mass distribution can be largely generalised to other mass distributions. The exception might be clusters with densities in excess of $\rho_{\text {core }}>10^{4} \mathrm{pc}^{-3}$ like NGC 3603 .

\section{References}

Aarseth, S. J. 1963, MNRAS, 126, 223

Aarseth, S. J. 1974, A\&A, 35, 237

Aarseth, S. J. 2003, Gravitational N-Body Simulations (Cambridge: Cambridge University Press)

Adams, F. C., Proszkow, E. M., Fatuzzo, M., \& Myers, P. C. 2006, ApJ, 641, 504

Akeson, R. L., Ciardi, D. R., van Belle, G. T., \& Creech-Eakman, M. J. 2002, ApJ, 566, 1124

Alexander, R. D., Clarke, C. J., \& Pringle, J. E. 2005, MNRAS, 358, 283

Alexander, R. D., Clarke, C. J., \& Pringle, J. E. 2006, MNRAS, 369, 229

Allen, P. R., Koerner, D. W., Reid, I. N., \& Trilling, D. E. 2005, ApJ, 625, 385

Andrews, S. M., \& Williams, J. P. 2007, ApJ, 659, 705

Balbus, S. A., \& Hawley, J. F. 2002, ApJ, 573, 749

Binney, J., \& Tremaine, S. 2008, Galactic Dynamics: Second Edition (Princeton University Press)

Boffin, H. M. J., Watkins, S. J., Bhattal, A. S., Francis, N., \& Whitworth, A. P. 1998, MNRAS, 300, 1189

Bonnell, I. A., \& Davies, M. B. 1998, MNRAS, 295, 691

Clarke, C. J., \& Pringle, J. E. 1993, MNRAS, 261, 190

Clarke, C. J., Gendrin, A., \& Sotomayor, M. 2001, MNRAS, 328, 485

Drake, J. J., Ercolano, B., Flaccomio, E., \& Micela, G. 2009, ApJ, 699, L35

Ercolano, B., Drake, J. J., Raymond, J. C., \& Clarke, C. C. 2008, ApJ, 688, 398

Fang, M., van Boekel, R., Bouwman, J., et al. 2013, A\&A, 549, A15

Figer, D. F., Kim, S. S., Morris, M., et al. 1999, ApJ, 525, 750

Gorti, U., \& Hollenbach, D. 2009, ApJ, 690, 1539

Hall, S. M. 1997, MNRAS, 287, 148

Hall, S. M., Clarke, C. J., \& Pringle, J. E. 1996, MNRAS, 278, 303

Heggie, D. C. 1975, MNRAS, 173, 729

Heller, C. H. 1993, ApJ, 408, 337

Heller, C. H. 1995, ApJ, 455, 252

Hillenbrand, L. A. 1997, AJ, 113, 1733

Hillenbrand, L. A., \& Hartmann, L. W. 1998, ApJ, 492, 540

Hillenbrand, L. A., Strom, S. E., Calvet, N., et al. 1998, AJ, 116, 1816

Hills, J. G. 1975, AJ, 80, 809

Johnstone, D., Matsuyama, I., McCarthy, I. G., \& Font, A. S. 2004, in Rev. Mex.

Astron. Astrofis. Conf. Ser. 22, eds. G. García-Segura, G. Tenorio-Tagle,

J. Franco, \& H. W. Yorke, 38

Kitamura, Y., Momose, M., Yokogawa, S., et al. 2002, ApJ, 581, 357

Klahr, H. H., \& Bodenheimer, P. 2003, ApJ, 582, 869 
M. Steinhausen and S. Pfalzner: Influence of disc-mass distribution on disc frequency in star clusters

Kley, W., Papaloizou, J. C. B., \& Ogilvie, G. I. 2008, A\&A, 487, 671

Kroupa, P. 1995, MNRAS, 277, 1491

Kroupa, P. 2001, MNRAS, 322, 231

Lada, C. J., Muench, A. A., Lada, E. A., \& Alves, J. F. 2004, AJ, 128, 1254

Larwood, J. D. 1997, MNRAS, 290, 490

Lay, O. P., Carlstrom, J. E., \& Hills, R. E. 1997, ApJ, 489, 917

Luhman, K. L., Allen, L. E., Allen, P. R., et al. 2008, ApJ, 675, 1375

Luhman, K. L., Mamajek, E. E., Allen, P. R., \& Cruz, K. L. 2009, ApJ, 703, 399

Matsuyama, I., Johnstone, D., \& Hartmann, L. 2003, ApJ, 582, 893

McCaughrean, M. J., \& Stauffer, J. R. 1994, AJ, 108, 1382

Moeckel, N., \& Bally, J. 2006, ApJ, 653, 437

Mundy, L. G., Looney, L. W., Erickson, W., et al. 1996, ApJ, 464, L169

Olczak, C., Pfalzner, S., \& Spurzem, R. 2006, ApJ, 642, 1140

Olczak, C., Pfalzner, S., \& Eckart, A. 2008, A\&A, 488, 191

Olczak, C., Pfalzner, S., \& Eckart, A. 2010, A\&A, 509, A63

Olczak, C., Kaczmarek, T., Harfst, S., Pfalzner, S., \& Portegies Zwart, S. 2012, ApJ, 756, 123

Ostriker, E. C. 1994, ApJ, 424, 292

Pfalzner, S. 2003, ApJ, 592, 986

Pfalzner, S. 2004, ApJ, 602, 356
Pfalzner, S., \& Kaczmarek, T. 2013, A\&A, 555, A135

Pfalzner, S., \& Olczak, C. 2007, A\&A, 462, 193

Pfalzner, S., \& Olczak, C. 2008, in IAU Symp. 246, eds. E. Vesperini, M. Giersz, \& A. Sills, 69

Pfalzner, S., Umbreit, S., \& Henning, T. 2005a, ApJ, 629, 526

Pfalzner, S., Vogel, P., Scharwächter, J., \& Olczak, C. 2005b, A\&A, 437, 967

Reipurth, B., Guimarães, M. M., Connelley, M. S., \& Bally, J. 2007, AJ, 134, 2272

Scally, A., \& Clarke, C. 2001, MNRAS, 325, 449

Scally, A., Clarke, C., \& McCaughrean, M. J. 2005, MNRAS, 358, 742

Shu, F. H., Adams, F. C., \& Lizano, S. 1987, ARA\&A, 25, 23

Steinhausen, M., Olczak, C., \& Pfalzner, S. 2012, A\&A, 538, A10

Stolte, A., Brandner, W., Brandl, B., Zinnecker, H., \& Grebel, E. K. 2004, AJ, 128,765

Stolte, A., Morris, M. R., Ghez, A. M., et al. 2010, ApJ, 718, 810

Tackenberg, J. 2009, Diploma Thesis, Univ. zu Köln

Testi, L., Natta, A., Shepherd, D. S., \& Wilner, D. J. 2001, ApJ, 554, 1087

Umbreit, S. 2001, Diploma Thesis, Univ. Jena

Wilner, D. J., Ho, P. T. P., Kastner, J. H., \& Rodríguez, L. F. 2000, ApJ, 534 L101 